\title{
Nested structures approach in designing an isotropic negative-index material for infrared
}

\author{
Andrei Andryieuski \\ andra@fotonik.dtu.dk \\ Radu Malureanu \\ rmal@fotonik.dtu.dk

\section{Andrei Lavrinenko} \\ alav@fotonik.dtu.dk
}

\begin{abstract}
DTU Fotonik Department of Photonics Engineering - Technical University of Denmark DTU-343, DK-2800, Kgs. Lyngby, Denmark

DTU Fotonik Department of Photonics Engineering - Technical University of Denmark DTU-343, DK-2800, Kgs. Lyngby, Denmark

DTU Fotonik Department of Photonics Engineering - Technical University of Denmark DTU-343, DK-2800, Kgs. Lyngby, Denmark
\end{abstract}

We propose a new generic approach for designing isotropic metamaterial with nested cubic structures. As an example, a three-dimensional isotropic unit cell design "Split Cube in Cage" (SCiC) is shown to exhibit an effective negative refractive index on infrared wavelengths. We report on the refractive index reaching -2.3 and the figure of merit as high as 2.7. The structure exhibits potential for application as a building block of isotropic negative-index materials. [D0I: 10.2971/jeos.2009.09003]

Keywords: negative index material, isotropic, metamaterial, infrared

\section{INTRODUCTION}

Metamaterials (MTMs) are artificially structured materials that can deliver unusual physical properties, never occurred in nature, to be used for our purposes. MTMs and especially double negative materials [1,2], which exhibit simultaneously negative electric permittivity $(\epsilon)$ and magnetic permeability $(\mu)$, attract a lot of attention in the scientific community because of the promising applications for superresolution imaging [3], nanocoupling [4], cloaking [5] etc.

The idea of double negative metamaterials have been realized with different implementations in microwave and optical regions: the split-ring resonator (SRR) [6] having negative magnetic permeability were combined with a wire medium [7] exhibiting negative electric permittivity; the fish-net structure [8] and cut-wire pairs [9] for negative refractive index in the infrared and visible regions. Several groups reported fabrication and characterization of negative-index materials (NIMs) [8]-[13]. Fabrication of such materials is still a challenging task thus triggering the development of new fabrication technologies [14]-[16].

One particular call is for isotropic MTMs, so a lot of efforts have been put into elaborating such materials [17]-[20]. It is well known that either cubic or random arrangement of the atoms (or meta-atoms) can lead to isotropy in properties [21]. There are two approaches to achieve this goal from the design point of view. The first one propose to arrange the SRR unit cells into a cubic lattice [22]. A frequency limitation of this design is due to the electromagnetic properties of metals [23]. The second approach suggests to exploit Mie resonances from dielectric spheres [24] arranged in the cubic lattice [25] or distributed randomly [26]. The material parameters needed to obtain the desired resonances in the optical regime can hardly be found, thus limiting again the application of such design by the microwave region.

In this paper we propose a generic approach for designing an isotropic NIM unit cell in the telecommunication wavelengths as a nested cubic structure. We will refer to our design as "Split Cube in Cage" (SCiC). By standard restoration procedure the effective parameters such as refractive index and impedance as well as permittivity and permeability are revealed and analyzed. We focus on parameters of the constitutive parts in separate and after their synthesis in the SCiC. Also we give the proof of its isotropy. Problems originated after combining several layers of such structure are highlighted in the concluding discussion.

\section{METHODOLOGY}

\subsection{NIM design}

The double negative material should in principle possess two parts, one providing a negative electric permittivity $(\epsilon<0)$ and the other responsible for a negative magnetic permeability $(\mu<0)$. The isotropy in optical properties claims high symmetry in the unit cell topology. To preserve the symmetry of the two parts we decided to nest one inside the other, like a cube in a cube. Each of the parts should contribute by its own resonance properties: either electric or magnetic.

It is well known that a medium consisting of long metallic wires gives negative permittivity [7]. It behaves effectively like a diluted metal, obeying the Drude-like frequency dependence. Having in mind the other part, which supposed to be inserted in the 3D metallic wire medium without any contacts 
between them, we are forced to move all wires onto the periphery of the unit cell. Thus design resembles a cage (Figure 1), and we will refer to it as "the Cage". The Cage (Figure 1) defines the unit cell border and consists of 6 connected perforated plates made from square cross-section wires having a thickness of $20 \mathrm{~nm}$ equidistantly placed along the edge of the unit cell. Each plate is of square shape with $250 \mathrm{~nm}$ length. The Cage possesses cubic symmetry, so should do the inner part of the cell.

Magnetic response can be generated by a split-ring resonator [6]. It is modified for plane isotropic response in [27]. We make further generalization extending it to a 3D design obeying cubic symmetry: the hollow cube. Resonance conditions are imposed by carving symmetrical slits in the centres of the cube facets (Figure 1). We will refer to the inner part of the unit cell as "Split Cube" (SCube). The SCube (Figure 1) is a metallic box $160 \mathrm{~nm}$ long, with $20 \mathrm{~nm}$ walls and $5 \mathrm{~nm}$ slits in the middle of the cube facets. The sizes of the Cage and SCube were obtained targeting the range close to $1.5 \mu \mathrm{m}$.

Both the SCube and the Cage are made from silver and embedded into silica (not shown for simplicity). In the simulations silver is described using the Drude model with plasma frequency $\omega_{p}=1.39 \cdot 10^{16} \mathrm{rad} / \mathrm{s}$ and collision frequency $v_{c}=1.68 \cdot 10^{13} \mathrm{~s}^{-1}[28]$.
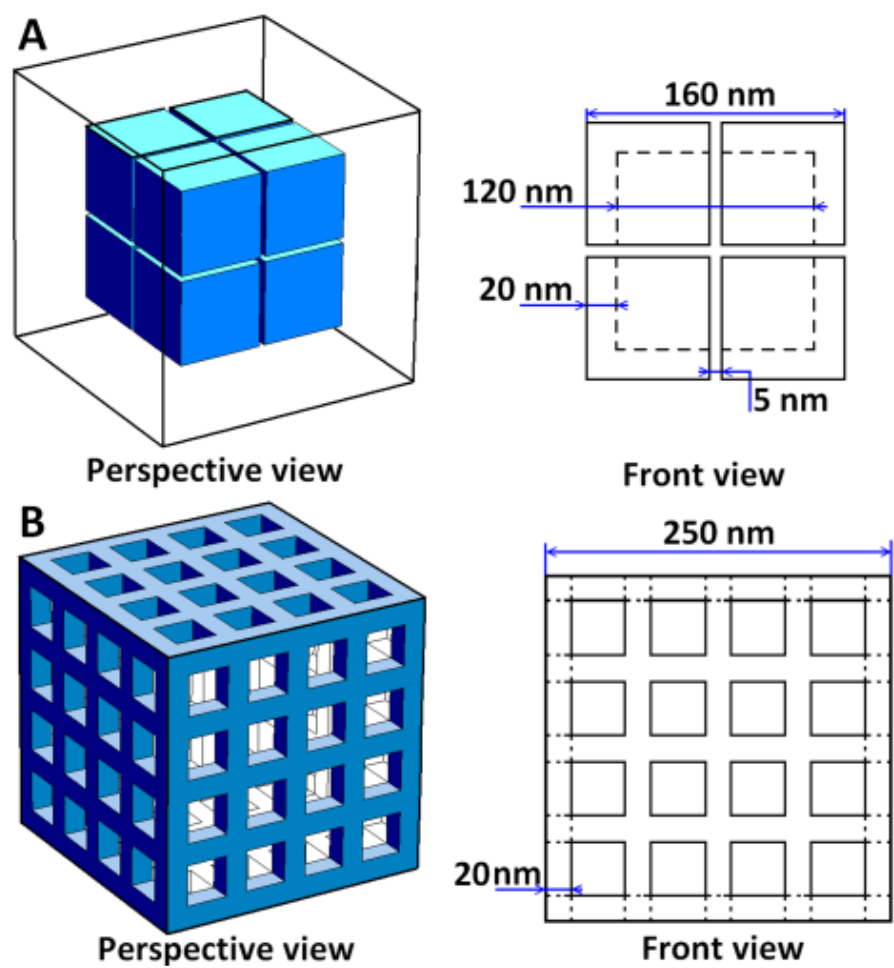

FIG. 1 Split Cube (A) and Cage (B) unit cell design parts

\subsection{Simulation method}

To characterize the structure and retrieve its effective parameters we simulated reflection and transmission amplitudephase spectra for normal incidence using CST Microwave Studio commercially available software [29]. As the unit cell size is $250 \mathrm{~nm}$ and the structure is embedded in silica, the highfrequency limit for this metamaterial to be regarded as homogenous medium is roughly about $200 \mathrm{THz}\left(a_{x}=a_{y}=\right.$ $\left.a_{z}=\lambda_{\text {silica }} / 4\right)$. So, in order to keep ourselves within the effective medium approximation simulations were performed in a frequency range from 20 to $220 \mathrm{THz}$. Periodic boundary conditions for the directions perpendicular to the propagation and open boundaries along the propagation direction were applied.

We restored the effective parameters (refractive index and impedance) using the conventional retrieval method for normal incidence [30]. According to [31] such parameters should be regarded as wave parameters, because they cannot provide complete description of the media, e.g. under the different angles of incidence.

\section{RESULTS}

\subsection{Cage}

The Cage acts like a diluted metal with reflectivity almost 1 and transmittivity lower than 0.01 (Figure 2). So, it is not surprisingly that restored effective permittivity (Figure 3) resembles the Drude model dispersion. However, the absolute

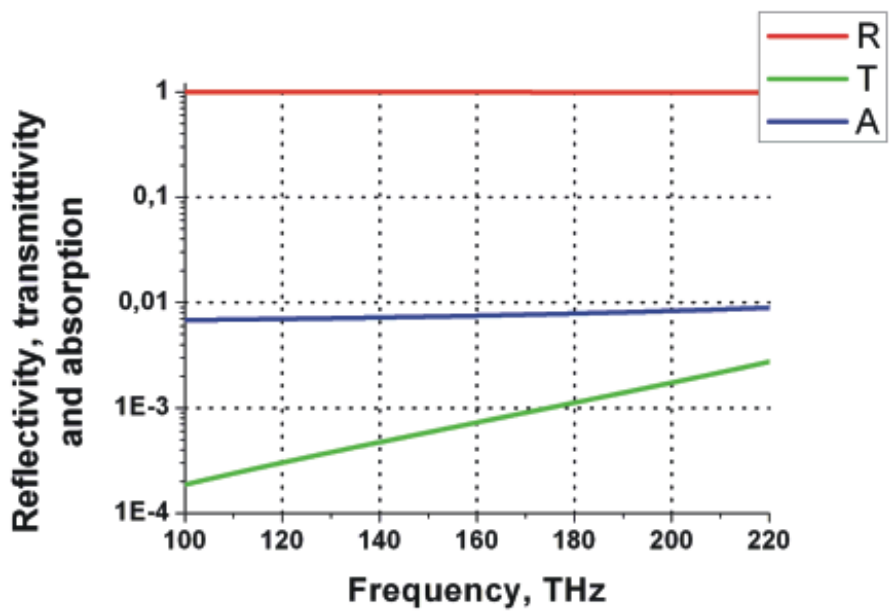

FIG. 2 Reflectivity (red), transmittivity (green) and absorption (blue) spectra of a one Cage layer

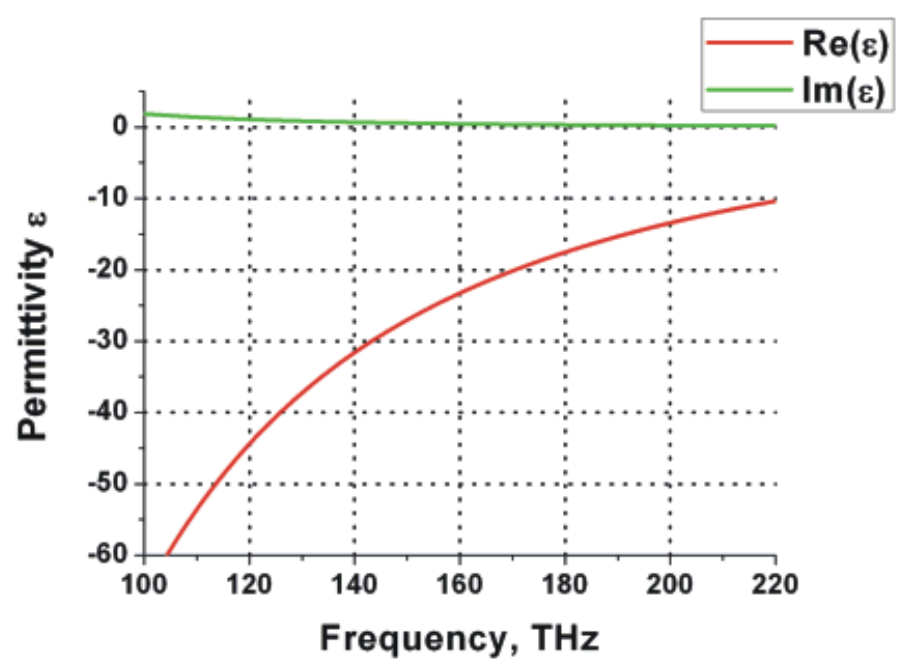

FIC. 3 Retrieved permittivity (red line is a real part and green - an imaginary part) spectrum of the Cage structure. This colour convention will be respected in all the subsequent figures 


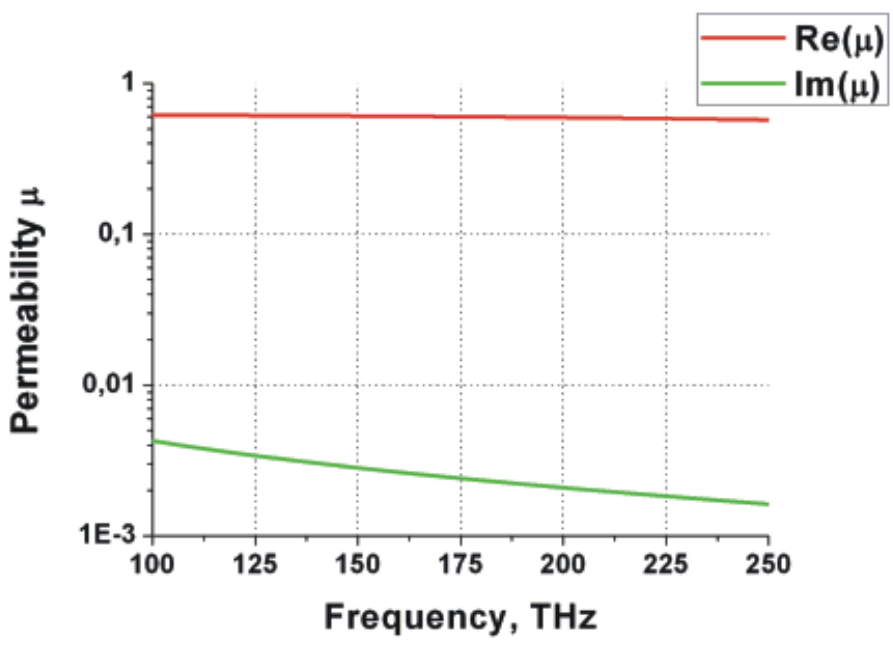

FIC. 4 Retrieved permeability spectrum of the Cage structure

value of permittivity is about 10 times smaller than for the bulk silver. Effective magnetic permeability (Figure 4) is less than 1.0 indicating a diamagnetic response [32].

\subsection{Split cube}

Transmittivity and reflectivity spectra of the SCube (Figure 5) show resonant behaviour around $175 \mathrm{THz}$. The SCube effectively acts like a 3D magnetic meta-atom providing negative magnetic permeability for the frequencies from 174.2 to $188.7 \mathrm{THz}$ (Figure 6). Thus it gives the possibility to obtain the negative refractive index. The minimum value of permeability is -0.62 . We note that a traditional antiresonance behaviour of permittivity (Figure 7) is observed in the region of negative permeability [33]. The negative value of $\operatorname{Im}(\epsilon)$ does not imply optical amplification, but rather energy transfer from magnetic to electric field (see discussion in [33]-[35]).

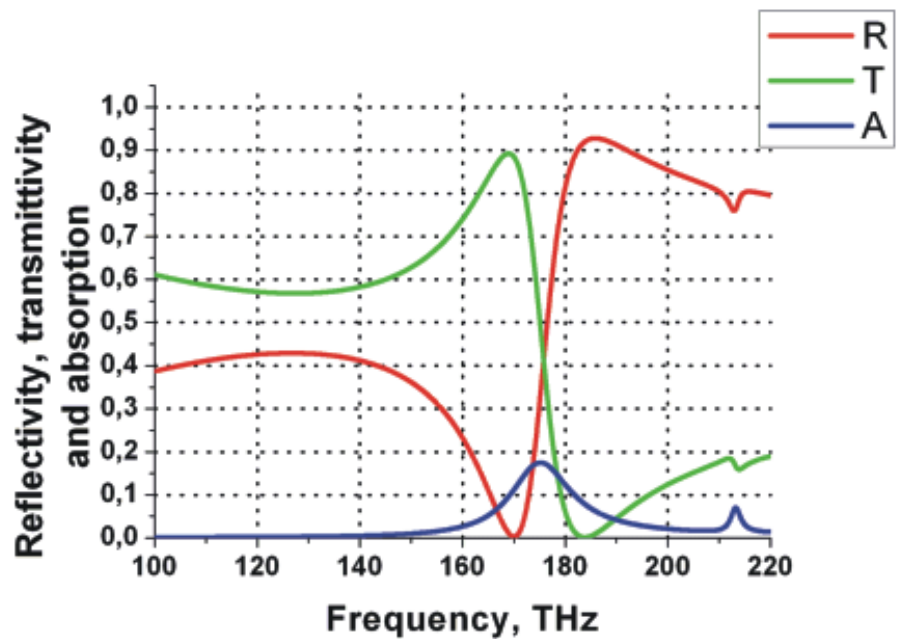

FIG. 5 Reflectivity (red), transmittivity (green) and absorption (blue) spectra of one layer of Scube

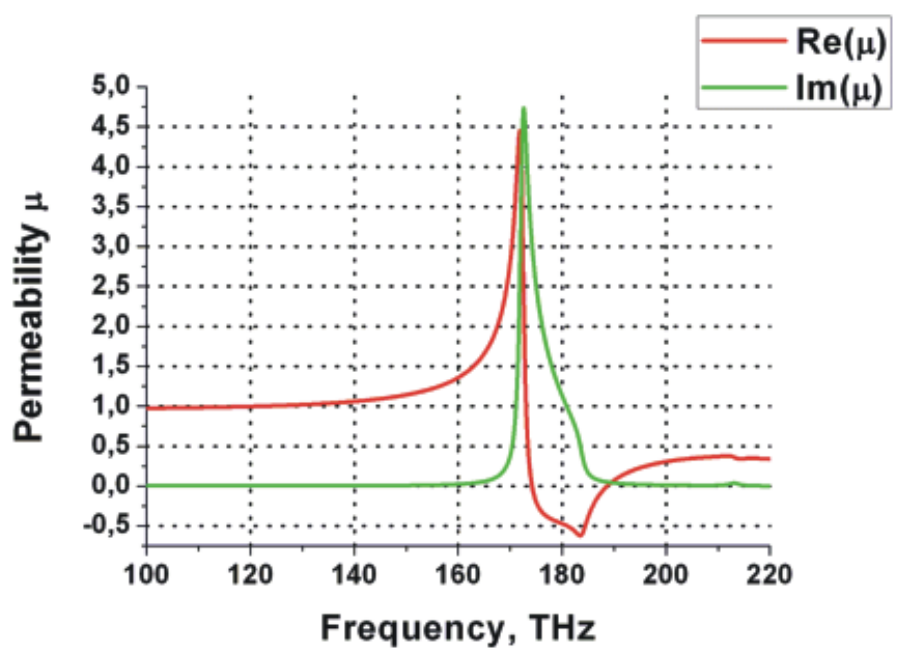

FIG. 6 Retrieved permeability spectrum of the SCube structure

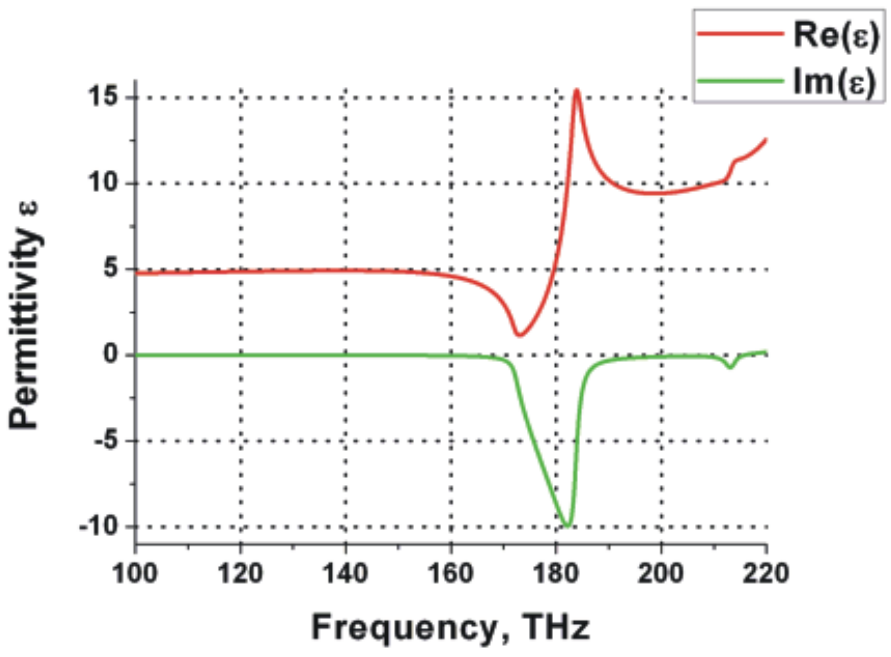

FIG. 7 Retrieved permittivity spectrum for the SCube structure

\subsection{Split cube in cage}

Inserting the SCube into the Cage we expect that as an outcome of their joint operation we will get the negative refractive index as in the other cases, e.g. split ring resonators with the wire medium, etc. However, the critical parameter is transmittivity, which could be quite low due to the Cage screening. Remarkably, the transmittivity spectrum of the one SCiC layer (Figure 8) shows two new peaks which are not observed for the cage and SCube separately (Figure 2 and Figure 5). The peak at $185.7 \mathrm{THz}$ (transmittivity 0.26) has high absorption (0.35), and rather high reflection (Figure 8). It corresponds to the region of negative refractive index (Figure 9). The second peak at 198.4 THz has very pronounced transmission with low absorption and negligible reflection. We will address the question about its origin in Section 4.

The refractive index is negative in the range from $f_{1}=$ $175.3 \mathrm{THz}$ to $f_{2}=191.8 \mathrm{THz}$ reaching its minimum value of -2.3 at $183.5 \mathrm{THz}$. The maximum figure of merit (FOM) is 2.7 and is shifted towards the edge of the absorption peak at $185.8 \mathrm{THz}$ (Figure 9). At this frequency the transmittivity is 0.26 , the absorption 0.33 while the refractive index has a value of -1.27 . 


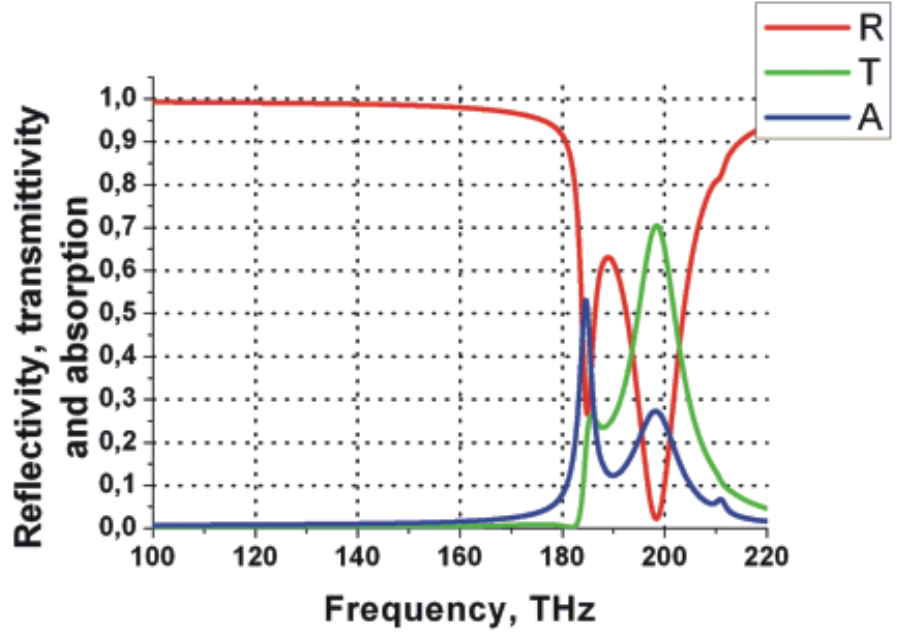

FIG. 8 Reflectivity (red), transmittivity (green) and absorption (blue) spectra of one layer of SCiC

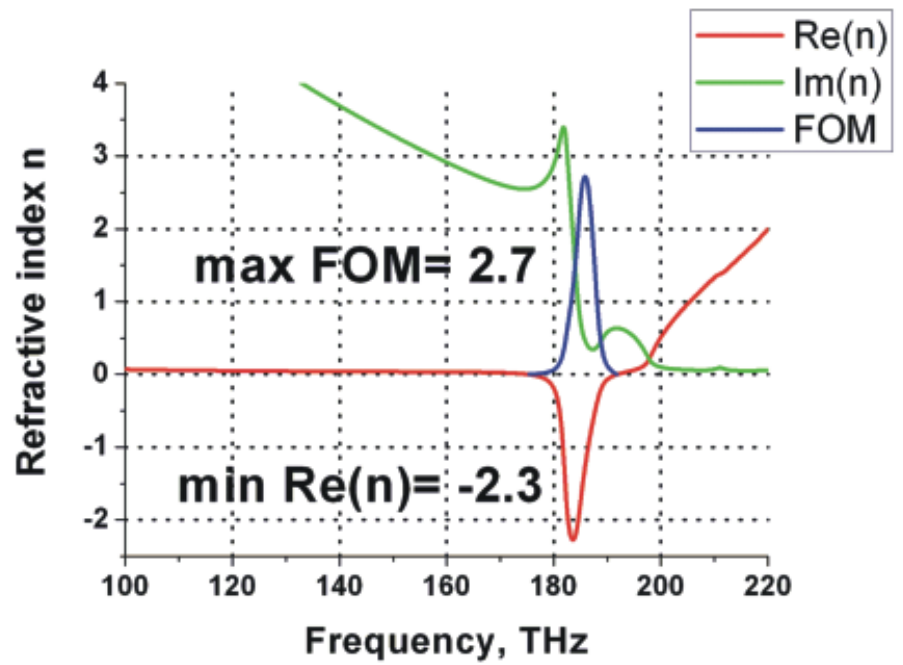

FIG. 9 Retrieved refractive index and figure-of-merit (blue) of one layer of SCiC

The restored effective parameters of the whole unit cell altered from both the Cage and SCube. A permittivity (Figure 10) has lower absolute value than for just the cage (Figure 3) and loses its negative value at around $200 \mathrm{THz}$. Still, its antiresonant behaviour is maintained at the frequency of the

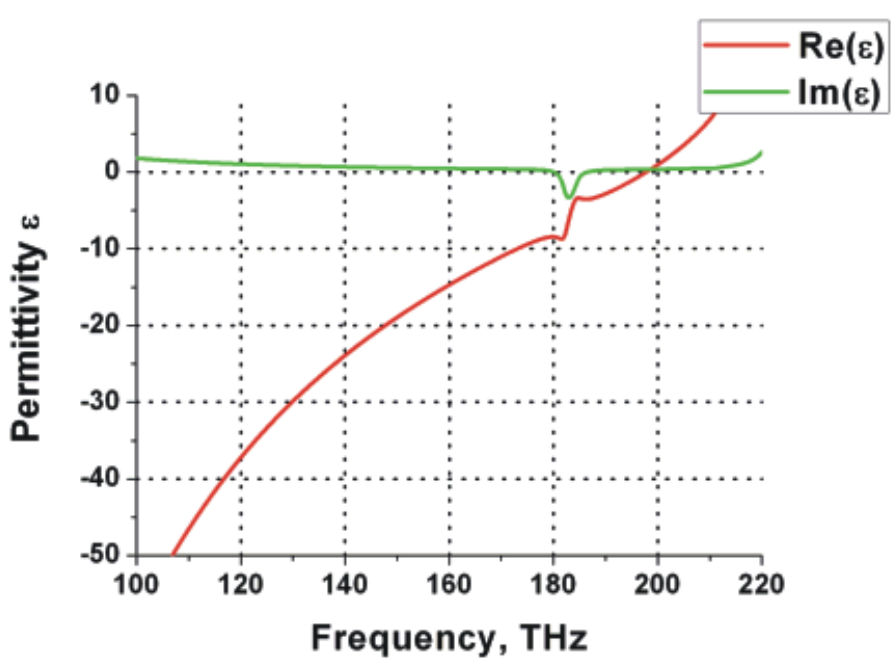

FIC. 10 Retrieved permittivity spectrum of one layer of SCiC

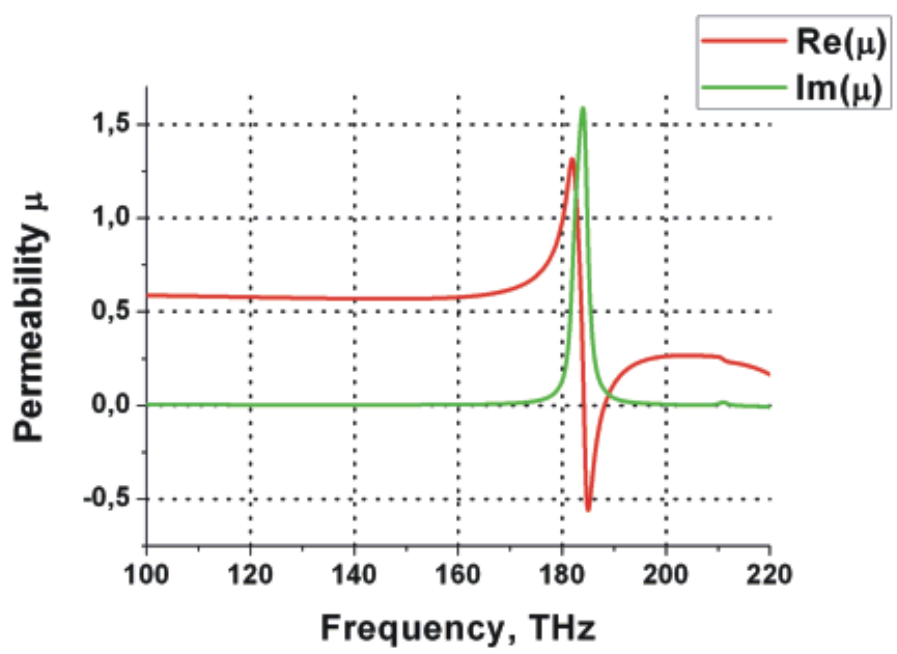

FIG. 11 Retrieved permeability spectrum of one layer of SCiC

magnetic resonance. A permeability (Figure 11) has almost the same minimum value but the more pronounced resonance, which leads to a narrower bandwidth compared with SCube (Figure 6).

\subsection{Several layers of SCiC}

It is important to check whether the NIM properties are preserved for the thicker structure. When the number of SCiC layers is increased the negative refractive index is still observed being shifted to lower frequencies and with lower absolute values (Figure 12).

Our explanation for his phenomenon is that the negative permeability value for several layers of $\mathrm{SCiC}$ is decreasing by the absolute value (Figure 13). However, the multiple layers of pure SCubes maintain the negative magnetic response (Figure 14) therefore, reduction in SCiCs magnetic response comes from the interaction between the Cage and the SCube structures. The negative value of the refractive index (Figure 12) is due to the high negative permittivity but, in this case, the losses are increased.

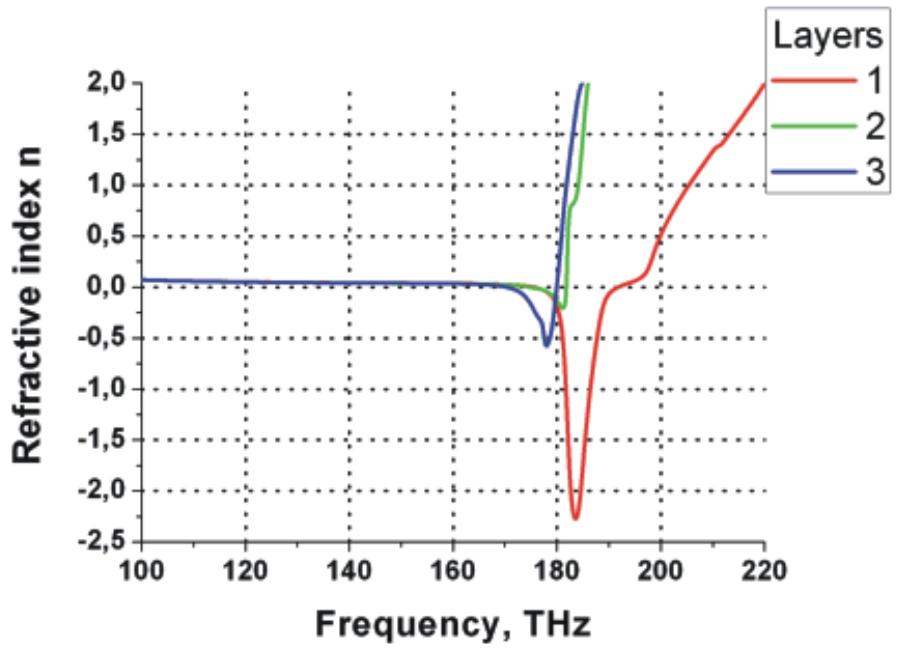

FIG. 12 Retrieved refractive index for one (red), two (green) and three (blue) layers of SCiC 


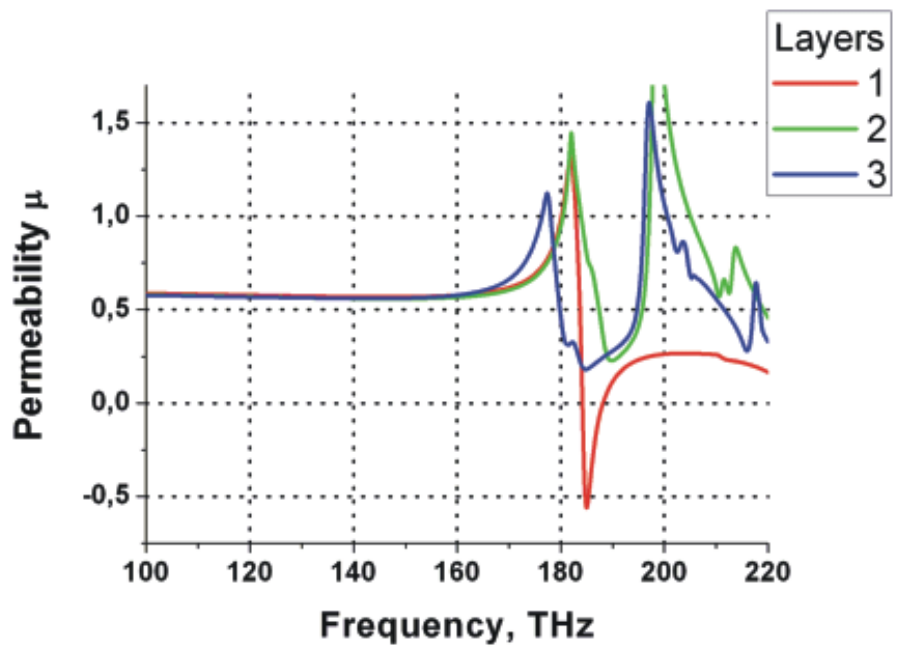

FIG. 13 Retrieved permeability for one (red), two (green) and three (blue) layers of SCiC

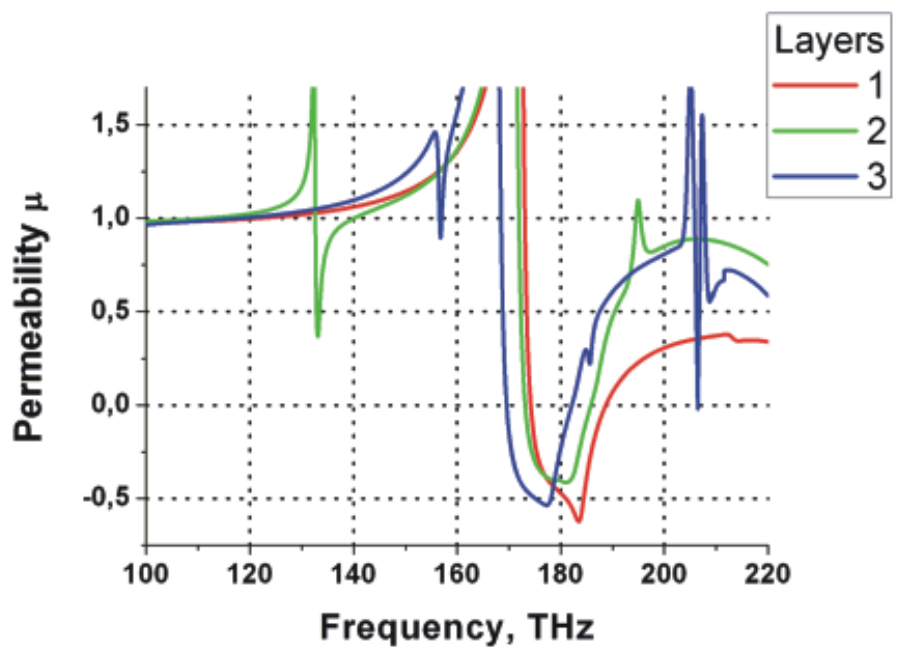

FIG. 14 Retrieved permeability for one (red), two (green) and three (blue) layers of Scube

\subsection{Band structure}

An ultimate prove of the structural isotropy is the band structure. If the eigenfrequencies have the same values for different directions in the reciprocal wave vector space, the material can be regarded as isotropic [17]. The band diagram is obtained using periodic boundaries in all spatial directions. To avoid the slow convergence generated by the losses in the metallic parts, we replace silver with a perfect electric conductor material. To be noted that this approach does not influence the isotropy or anisotropy of the structure, but changes the eigenfrequencies values. The perfect electric conductor reduces the effective volume of the unit cell accessible to fields that pushes eigenfrequencies to higher values.

We calculated band diagram of the proposed NIM for $\Gamma-X, \Gamma-$ $\mathrm{M}$ and $\Gamma$-R directions (Figure 15). The homogenization procedure is valid for long wavelengths, or for small wavevectors. So, we are interested in the region close to $k=0$. Here the eigenfrequencies are almost the same for all aforementioned directions. In addition all the bands are nearly flat. The latter is an evidence of the significant reduction of the spatial dispersion.

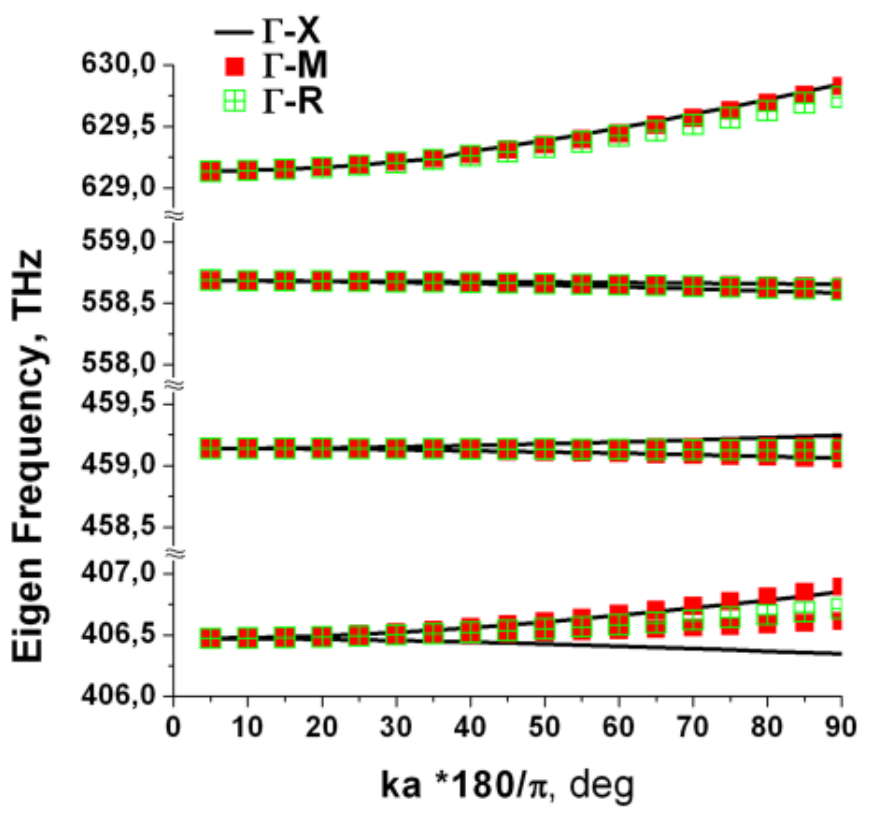

FIC. 15 Band structure of the Split-Cube in Cage made from perfect electric conductor (black line $-\Gamma-\mathrm{X}$, red squares $-\Gamma-\mathrm{M}$, green crosses $-\Gamma-\mathrm{R}$ )

\section{DISCUSSION}

As we see from Figure 10 and 11 effective permittivity and permeability of the one layer of the Cage and the SCube behave as expected: the Cage gives negative permittivity and the SCube gives negative permeability. The SCube has also minor influence on permittivity which becomes apparent through its antiresonance. In the same time the Cage has a diamagnetic response perturbing magnetic properties of the SCiC structure. Nevertheless, these effects cannot overwhelm the principle ones.

The proposed NIM with a nested structure has a simple cubic lattice what is required for being isotropic and its unit cell respects all the symmetry relations in ordered to be qualified for an isotropic structure [36]. The isotropy of the proposed design is confirmed by the band diagram (Figure 15). The bands are almost flat showing reduced spatial dispersion. Such behaviour was reported in the investigation of a 2D photonic crystal structure between two perfect conducting plates [37]. Moreover, as it was shown in [38], the effective recipe to reduce the pronounced spatial dispersion of the metallic wire medium is to insert additional metallic plates or small cubes.

The utilized restoration procedure by default is oriented on the simplest case of material equations in the effective medium. We should eliminate more sophisticate cases like biisotropic medium, when in addition to permittivity $\epsilon$ and permeability $\mu$ two more complex parameters $\xi$ and $\zeta$ appear [39]. But as our unit cell (Figure 1) possesses inversion symmetry, parameters of biisotropy vanish [36, 40], thus we can characterize the structure using $\epsilon$ and $\mu$ values only.

It is interesting to observe that $\mathrm{SCiC}$ transmission is higher than the product of the Cage and the SCube transmissions. There are two high transmittivity peaks at $185.7 \mathrm{THz}$ and 198.4 THz (Figure 8). Since these peaks are hardly visible for 
the two design elements in separate (Figure 2 and Figure 5 respectively) we suppose that they are due to the interaction between the parts. It is unlikely that this is a plasmon-induced interaction because the periodicity of SCiC structure and its features sizes corresponds to the plasmon frequencies in the visible range [41]. We attribute this phenomenon to the FabryPerot resonance with the main peak at $198.4 \mathrm{THz}$ overlapping with the resonant SCube behaviour at $185.7 \mathrm{THz}$. The signature of the Fabry-Perot resonances with inhibited reflection might be recognized at $198.4 \mathrm{THz}$. However, detailed understanding requires special investigation.

In case of few cells stacked in the direction of propagation the problem of loosing magnetic response of the combined structure rises up. In addition, fields in the subsequent layers show fast attenuation. We think that this problem can be solved by structural design optimization, so further analysis of the nested structures properties is needed.

\section{CONCLUSION}

In this paper we discussed a new prospective NIM design for isotropic metamaterial application. Our idea shows a generic approach. In principle nested structural elements can differ from cubes and cages. The one particular example we focus ourselves is the Split Cube in Cage. One layer of the SCiC possesses effective refractive index having a minimum value of -2.3 and maximum figure-of-merit of 2.7 in the band. It is composed by two distinct parts, each contributing to the overall negative response. The SCube gives negative permeability while the Cage is responsible for negative permittivity. Band structure analysis and unit cell symmetry confirm that such NIM is isotropic. The questions of high transmittivity at some frequencies and loss of negative refractive index for several layers of SCiC require further investigation.

\section{ACKNOWLEDGEMENTS}

We gratefully acknowledge partial support from the Danish Research Council for Technology and Production Sciences via the NIMbus project.

\section{References}

[1] V. Veselago, "The electrodynamics of substances with simultaneously negative values of $\epsilon$ and $\mu$ " Sov. Phys. Uspekhi. 10, 509-514 (1968).

[2] D. R. Smith, W. J. Padilla, D. C. Vier, S. C. Nemat-Nasser, and S. Schultz, "Composite medium with simultaneously negative permeability and permittivity" Phys. Rev. Lett. 84, 4184-4187 (2000).

[3] J. B. Pendry, "Negative refraction makes a perfect lens" Phys. Rev. Lett. 85, 3966-3969 (2000).

[4] A. Degiron, D. R. Smith, J. Mock, B. Justice, and J. Gollub, "Negative index and indefinite media waveguide couplers" Appl. Phys. 87, 321-328 (2007).

[5] D. Schurig, J. Mock, B. Justice, S. Cummer, J. B. Pendry, A. vStarr, and D. R. Smith, "Metamaterial electromagnetic cloak at microwave frequencies" Science 314, 977-980 (2006).
[6] J. B. Pendry, A. Holden, D. Robbins, and W. Stewart, "Magnetism from conductors and enhanced nonlinear phenomena" IEEE T. Microw. Theory. 47, 2075-2084 (1999).

[7] W. Rotman, "Plasma simulation by artificial dielectrics and parallel-plate media" IRE Trans. Antennas Propag. AP-10, 82-95 (1962).

[8] S. Zhang, W. Fan, K. Malloy, S. Brueck, N. Panoiu, and R. Osgood, "Near-infrared double negative materials" Opt. Express 13, 49224930 (2005).

[9] V. Shalaev, W. Cai, U. Chettiar, H. Yuan, A. Sarychev, V. Drachev, and $A$. Kildishev, "Negative index of refraction in optical metamaterials" Opt. Lett. 30, 3356-3358 (2005).

[10] V. Lomakin, Y. Fainman, Y. Urzhumov, and G. Shvets, "Doubly negative metamaterials in the near infrared and visible regimes based on thin film nanocomposites" Opt. Express 14, 11164-11177 (2006).

[11] C. M. Soukoulis, S. Linden, and M. Wegener, "Negative refractive index at optical wavelengths" Science 315, 47-49 (2007).

[12] H. Lezec, J. Dionne, and H. Atwater, “Negative refraction at visible frequencies" Science 316, 430-432 (2007).

[13] C. Garcia-Meca, R. Ortuño, R. Salvador, A. Martinez, and J. Marti, "Low-loss single-layer metamaterial with negative index of refraction at visible wavelengths" Opt. Express 15, 9320-9325 (2007).

[14] J. Valentine, S. Zhang, T. Zentgraf, E. Ulin-Avila, D. Genov, G. Bartal, and X. Zhang, "Three-dimensional optical metamaterial with a negative refractive index" Nature 455, 376-379 (2008).

[15] M. Rill, C. Plet, M. Thiel, I. Staude, G. Freymann, S. Linden, and M. Wegener, "Photonic metamaterials by direct laser writing and silver chemical vapour deposition" Nat. Mater. 7, 543-546 (2008).

[16] A. Boltasseva and V. Shalaev, "Fabrication of optical negativeindex metamaterials: recent advances and outlook" Metamaterials 2, 1-17 (2008).

[17] A. Iyer and G. Eleftheriades, "A three-dimensional isotropic transmission-line metamaterial topology for free-space excitation" Appl. Phys. Lett. 92, 261106 (2008).

[18] C. Caloz and T. Itoh, Electromagnetic Metamaterials: transmission line theory and microwave applications (Hoboken: Wiley Interscience, 2006)

[19] N. Landy, C. Bingham, T. Tyler, N. Jokerst, D. R. Smith, and W. Padilla, "Design, theory, and measurement of a polarization insensitive absorber for terahertz imaging" arXiv:0807.3390v2 [condmat.mtrl-sci], (2008).

[20] K. Alici and E. Ozbay, "A planar metamaterial: Polarization independent fishnet structure" Photonics Nanostruct. 6, 102-107 (2008).

[21] M. Born and E. Wolf, Principles of Optics (Cambridge: Cambridge University Press, 1999)

[22] T. Koschny, L. Zhang, and C. M. Soukoulis, "Isotropic threedimensional left-handed metamaterials" Phys. Rev. B. 71, 121103 (2005).

[23] M. Klein, C. Enkrich, M. Wegener, C.M. Soukoulis, and S. Linden, "Single-slit split-ring resonators at optical frequencies: limits of size scaling" Opt. Lett. 31, 1259-1261 (2006).

[24] V. Yannopapas and A. Moroz, "Negative refractive index metamaterials from inherently non-magnetic materials for deep infrared to terahertz frequency range" Condens Matter Phys.17, 3717-3734 (2005).

[25] I. Vendik, 0. Vendik, and M. Odit, "Isotropic artificial media with 
simultaneously negative permittivity and permeability" Microw. Opt. Techn. Let. 48, 2553-2556 (2006).

[26] A. Kussow, A. Akyurtlu, and N. Angkawisittpan, "Optically isotropic negative index of refraction metamaterial" Phys. Status. Solidi. B. 245, 992-997 (2008).

[27] R. Penciu, K. Aydin, M. Kafesaki, T. Koschny, E. Ozbay, E. Economou, and C. M. Soukoulis, "Multi-gap individual and coupled split-ring resonator structures" Opt. Express 16, 18131-18144 (2008).

[28] J. Gordon and R. Ziolkowski, "The design and simulated performance of a coated nano-particle laser" Opt. Express 15, 2622-2653 (2007).

[29] CST Microvawe Studio, http://www.cst.com/

[30] D. Smith, S. Schultz, P. Markos, and C. Soukoulis, "Determination of effective permittivity and permeability of metamaterials from reflection and transmission coefficients" Phys. Rev. B. 65, 195104 (2002).

[31] C. Menzel, C. Rockstuhl, T. Paul, F. Lederer, and T. Pertsch, "Retrieving effective parameters for metamaterials at oblique incidence" Phys. Rev. B. 77, 195328 (2008).

[32] E. Economou, T. Koschny, and C. Soukoulis, "Strong diamagnetic response in split-ring-resonator metamaterials: Numerical study and two-loop model" Phys. Rev. B. 78, 92401 (2008).

[33] T. Koschny, P. Markos, D.R. Smith, and C.M. Soukoulis, “Resonant and antiresonant frequency dependence of the effective parameters of metamaterials" Phys. Rev. E. 68, 65602 (2003).

[34] R. Depine and A. Lakhtakia, "Comment I on: Resonant and antiresonant frequency dependence of the effective parameters of metamaterials" Phys. Rev. E. 70, 048601 (2004).

[35] A. Efros, "Comment II on: Resonant and antiresonant frequency dependence of the effective parameters of metamaterials" Phys. Rev. E. 70, 048602 (2004).

[36] J. Baena, L. Jelinek, and R. Marqués, “Towards a systematic design of isotropic bulk magnetic metamaterials using the cubic point groups of symmetry" Phys. Rev. B. 76, 245115 (2007).

[37] A. Maradudin and A. McGurn, "Photonic band structure of a truncated, two-dimensional, periodic dielectric medium" J. Opt. Soc. Am. B. 10, 307-313 (1993).

[38] A. Demetriadou and J. Pendry, "Taming spatial dispersion in wire metamaterial" J. Phys. 20, 295222 (2008).

[39] J. A. Kong, Electromagnetic Wave Theory (New York: Wiley. 1986)

[40] T. Koschny, L. Zhang, and C. M. Soukoulis, "Isotropic threedimensional left-handed metamaterials" Phys. Rev. B. 71, 121103 (2005).

[41] T. Ebbesen, H. Lezec, H. Ghaemi, T. Thio, and P. Wolff, "Extraordinary optical transmission through sub-wavelength hole arrays" Nature 391, 667 (1998). 